\title{
Nanoscale
}

\section{Micromotor-based lab-on-chip immunoassays $†$}

Cite this: Nanoscale, 2013, 5, 1325

Received 21st August 2012

Accepted 11th October 2012

DOI: $10.1039 / \mathrm{c} 2 \mathrm{nr} 32400 \mathrm{~h}$

www.rsc.org/nanoscale

\author{
Miguel García, $\ddagger^{\mathrm{ab}}$ Jahir Orozco, $\neq^{\mathrm{a}}$ Maria Guix, $\neq^{\mathrm{ac}}$ Wei Gao, ${ }^{\mathrm{a}}$ \\ Sirilak Sattayasamitsathit, ${ }^{a}$ Alberto Escarpa, ${ }^{b}$ Arben Merkoçi ${ }^{\mathrm{c}}$ and Joseph Wang ${ }^{* a}$
}

Here we describe the first example of using self-propelled antibody-functionalized synthetic catalytic microengines for capturing and transporting target proteins between the different reservoirs of a labon-a-chip (LOC) device. A new catalytic polymer/Ni/Pt microtube engine, containing carboxy moieties on its mixed poly(3,4-ethylenedioxythiophene) (PEDOT)/COOH-PEDOT polymeric outermost layer, is further functionalized with the antibody receptor to selectively recognize and capture the target protein. The new motor-based microchip immunoassay operations are carried out without any bulk fluid flow, replacing the common washing steps in antibody-based protein bioassays with the active transport of the captured protein throughout the different reservoirs, where each step of the immunoassay takes place. A first microchip format involving an 'on-the-fly' double-antibody sandwich assay (DASA) is used for demonstrating the selective capture of the target protein, in the presence of excess of non-target proteins. A secondary antibody tagged with a polymeric-sphere tracer allows the direct visualization of the binding events. In a second approach the immuno-nanomotor captures and transports the microsphere-tagged antigen through a microchannel network. An anti-protein-A modified microengine is finally used to demonstrate the selective capture, transport and convenient label-free optical detection of a Staphylococcus aureus target bacteria (containing proteinA in its cell wall) in the presence of a large excess of non-target (Saccharomyces cerevisiae) cells. The resulting nanomotor-based microchip immunoassay offers considerable potential for diverse applications in clinical diagnostics, environmental and security monitoring fields.

\section{Introduction}

The reliable identification, isolation and quantification of proteins holds an enormous potential to satisfy the growing demands in diverse fields, including medical diagnostics, food safety, forensic analysis, environmental monitoring and biodefense applications. ${ }^{1} \mathrm{~A}$ wide variety of techniques are available for protein detection and purification. Particularly attention has been given to antibody-based standard ELISA protocols within multiple well plates, ${ }^{2,3}$ although such assays often require long incubation times and multiple washing steps. Recent advances in the field of microanalytical systems have led to the development of lab-on-a-chip (LOC) immunoassays, coupling the power of microchip devices with the high specificity and sensitivity of antigen-antibody interactions. ${ }^{4-8}$ Yet, new efficient microchip

\footnotetext{
${ }^{a}$ Department of Nanoengineering, University of California-San Diego, La Jolla, CA 92093,USA. E-mail: josephwang@ucsd.edu

${ }^{b}$ Department of Analytical Chemistry and Chemical Engineering, University of Alcalá, E-28871 Alcalá de Henares, Madrid, Spain

'ICREA \& Catalan Institute of Nanotechnology, Campus University Autónoma de Barcelona, E-08193 Bellaterra, Barcelona, Spain

$\dagger$ Electronic supplementary information (ESI) available. See DOI: $10.1039 / \mathrm{c} 2 \mathrm{nr} 32400 \mathrm{~h}$

$\ddagger$ These authors have contributed equally to this work.
}

immunoassays offering direct, simple and rapid isolation of target proteins are desired.

Here we present a novel 'on-the-fly' microchip sandwich immunoassay based on self-propelled antibody-functionalized artificial microtransporters able to perform each of the immunoassay steps in different reservoirs and providing direct visualization of the protein binding event. Biological motors and related motion-driven processes have demonstrated the ability to capture and transport target proteins along microtubule tracks in microchip devices. ${ }^{9-11}$ One attractive example is a kinesin-powered 'smart dust' sensing device, containing antibody-functionalized microtubules, where the antigen capture and transport allow the replacement of the common washing steps. ${ }^{11}$ Microtubule-based molecular shuttles, coupled to kinesin-coated microchannel tracks, were also used for motionbased protein sorting. ${ }^{9,10}$ However, practical utility of such kinesin-based microchip immunoassays is hindered by the limited operational stability and lifetime of biological motors in engineering environments (associated with the rapid degradation of proteins outside biological environment) and by the necessity of preconfigured microlithographic tracks to guide their movement. ${ }^{9,11}$

The limitations of protein nanomotors have motivated the present study in which self-propelled antibody-functionalized artificial microtransporters have been incorporated for the first 
time into microchannel networks to create an advanced autonomous microchip device that integrates the protein capture, transport and detection operations within different separated zones. Synthetic catalytic nanomotors ${ }^{\mathbf{1 2 - 1 7}}$ are highly compatible with engineered microsystems and environments and can thus be readily adapted to different LOC formats. ${ }^{18}$ Non-functionalized nanowire and microtube motors were used earlier for a guided movement and cargo-towing within complex microchannel networks of microchip devices. ${ }^{\mathbf{1 9 2 0}}$ Receptorfunctionalized artificial nanomotors can offer the selective isolation of biological targets from raw physiological fluids by capturing and transporting them to a clean environment. ${ }^{21}$ While several self-propelled microtransporters have thus recently been described for isolating different biological targets, ${ }^{22-25}$ there are no reports on the use of artificial micromotors for antibody-based protein immunoassays and related LOC protocols.

In the following sections we will demonstrate the first example of a LOC immunoassay based on the active transport of antibody-functionalized synthetic micromotors (Fig. 1). As illustrated in Fig. 1, A, a simplified preparation of the functionalized microtransporters has been performed, relying on the presence of carboxy-terminated outermost polymeric layer

Mine surface modification

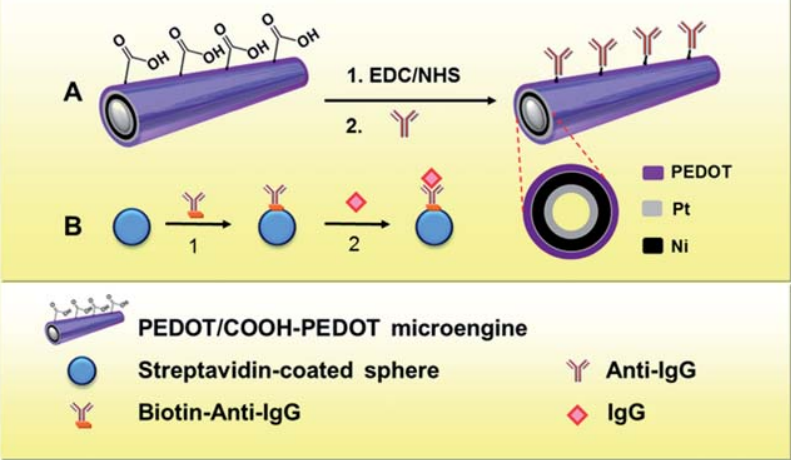

II. Microchip protein isolation DASA Sphere-tagged antigen
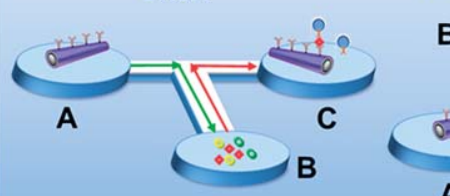

B 8
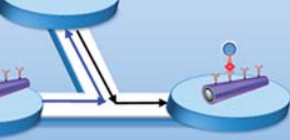

A

Fig. 1 Schematic of the micromotor-based microchip immunoassay. Microengine surface modification (I): immobilization of anti-lgG on the $\mathrm{COOH}-$ PEDOT:PEDOT microengine surface (A). Activation of the $\mathrm{COOH}$ groups through EDC/NHS chemistry (1) and immobilization of the anti-IgG antibody on the active moieties (2). Modification of streptavidin-coated PP (B) with the biotinylated-antiIgG antibody (1) and IgG antigen (2). Micromotor-based microchip immunoassay (II): in a DASA format, the anti-IgG-modified microengines are introduced into reservoir $A$ and guided towards reservoir $B$, where they navigate for $10 \mathrm{~min}$ in a solution containing the target protein in the presence 10 -fold non-target proteins. The modified microengine with the target protein is conducted to a third reservoir containing the tagged-PP. In a sphere tagged-antigen format, the anti-IgG-modified microengines are introduced in reservoir $A$ and guided towards a reservoir $B$ to pick-up the anti-IgG-modified PP and guided to reservoir $C$. of the template-deposited polymer/Ni/Pt microtubes. The resulting antibody-functionalized micromotors offer an 'on-thefly' capture and transport of the target antigen and, in a second step, the pickup of a sphere-tagged secondary antibody, thus demonstrating the efficiency of the different conjugation events held in each microchip reservoirs (Fig. 1, II left). Transport step thus replaces the washing steps common in traditional doubleantibody sandwich assays (DASA), in a manner analogous to kinesin-based 'smart dust' immunoassays. ${ }^{11}$ Tagging the secondary antibody with the polymeric sphere offers a convenient direct visualization and detection of the immunoreactions. In a second approach, shown in Fig. 1, II (right), the functionalized microengine captures and transports the microsphere-tagged antigen through a microchannel network. Therefore, convenient optical visualization of the detection and transport events related to Staphylococcus aureus ( $S$. aureus), used as target bacteria which contains proteinA in its cell wall, is also achieved by using anti-proteinA antibody-functionalized microengines. Such marriage of immunoassays, self-propelled artificial microtransporters, motion-based direct detection and microchip devices holds considerable promise for diverse applications of lab-on-a-chip systems.

\section{Results and discussion}

The new immuno-microtransporters were prepared by a template-based electrodeposition of multilayer PEDOT:COOHPEDOT/Ni/Pt microtubes. Fig. 1 depicts the surface modification of the microengine (I) along with a schematic of the tubular microtransporter and its corresponding cross-section. The outermost polymeric layer is synthesized by co-electropolymerization of PEDOT:PEDOT-COOH (from an electroplating solution containing an equimolar mixture of the monomers) followed by electrodeposition of the $\mathrm{Ni}$ and $\mathrm{Pt}$ metallic layers (from a Pt-Ni mixture electroplating solution). Whereas the inner Pt catalytic layer of the microtransporter is essential for generating the oxygen bubble thrust, the intermediate $\mathrm{Ni}$ layer is used for the magnetic guidance. The outermost PEDOT:COOH-PEDOT layer serves to anchor the antibody through the common carbodiimide (EDC)/ $N$-hydroxysuccinimide (NHS) chemistry (Fig. 1, A). Initial efforts involving a single $\mathrm{COOH}-\mathrm{PEDOT}$ external layer resulted with numerous $\mathrm{COOH}$ groups exposed on the microtransporter outermost surface for subsequent functionalization step. However, the codeposition of a COOH-PEDOT:PEDOT was proved necessary to avoid adherence of the PEDOT-based microtransporters to the surface of a glass slide after the antibody functionalization. Electrochemical deposition conditions, such as composition of the electroplating solutions, applied current, potential and deposition time, were optimized (Table S1†; other optimal conditions are described in the Experimental section). Carboxy moieties from the electropolymerized PEDOT:COOH-PEDOT external layer allow a direct functionalization of the resulting microengines, thereby obviating the need for e-beam deposition process of an outer gold layer and related clean room facilities. Unlike other microtubular engines, whose speed is greatly reduced after the e-beam gold deposition and further surface 
functionalization, the fast original speed of the $\mathrm{COOH}-$ PEDOT:PEDOT/Ni/Pt microtransporters $\left(\sim 400 \mu \mathrm{m} \mathrm{s}^{-1}\right)$ is not affected by this direct functionalization process. Furthermore, eliminating the need for self-assembled monolayers of alkanethiols ${ }^{25,26}$ obviates potential poisoning of the inner Pt surface, hence ensuring high catalytic activity and therefore the efficient microtransporters movement. It should be noted that unlike earlier gold-based microengine functionalization schemes, involving modification of half of the outer surface, ${ }^{25,26}$ the present protocol allows the functionalization of the entire outer surface, as the carboxy moieties cover the entire outermost polymeric surface that will be coupled later to the antibody receptor.

The resulting polymer/Ni/Pt microtransporters propel efficiently via the expulsion of oxygen bubbles generated by the catalytic oxidation of hydrogen peroxide fuel at their inner Pt layer. ${ }^{27,28}$ The template fabrication process results in $8 \mu \mathrm{m}$-long microtubes, similar to the scale of the particle-microsphere tag, which offers a convenient real-time optical visualization of the protein binding event. For example, the formation and movement of the immuno-sandwich complex can be readily visualized by tagging the secondary antibody with a microsphere tracer. Fig. 1, II illustrates the micromotor-based microchip immunoassay protein isolation protocol. All the capture-transport-tag-transport steps involved in the immunoassay protocol are thus carried out in the microfluidic device, hence replacing the capture-wash-tag-wash sequence common in conventional DASA protocols.

The ability of the micromotor to travel within a predetermined path along the microchip channels is crucial for the successful performance of the new LOC immunoassay protocol. Fig. 2 and Video $\mathrm{S} 1 \mathrm{~A} \dagger$ show the guided movement of the unmodified polymer/Ni/Pt microengine within different sections of the LOC microchannel network containing a PBS solution along with the $\mathrm{H}_{2} \mathrm{O}_{2}$ fuel and sodium cholate ( $\mathrm{NaCh}$ ) surfactant. These images demonstrate the self-propelled micromotor departing from the reservoir (a), crossing the channel interconnecting reservoirs (b) and entering the T-shape cross-section while swimming within this junction (c). The generated oxygen bubbles are clearly noticeable during such autonomous movement inside the microchip, as previously reported for other microengines. ${ }^{20,23}$ As indicated from the corresponding video, the microengine displays an efficient
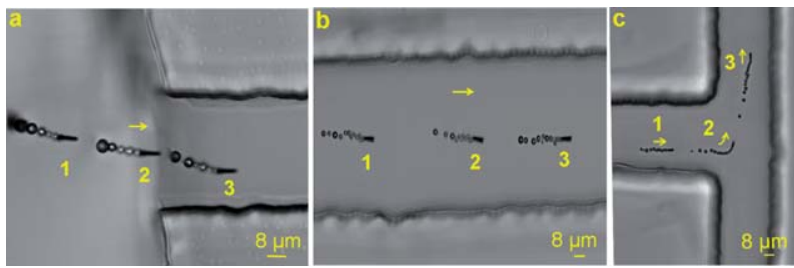

Fig. 2 Navigation of an unmodified $\mathrm{COOH}-\mathrm{PEDOT}$ :PEDOT microengine within a LOC device. Different overlaid time lapse images (labeled as 1, 2, 3) of the microengine leaving a reservoir (a), crossing the channel between two reservoirs (b) and entering the T-shape cross-section (c). Arrows indicate the direction of the movement. movement and advanced spatial motion control within the narrow channels and the different reservoirs in the microchip, reflecting the precise magnetic guidance associated with its intermediate Ni layer. Such advanced guided motion and efficient propulsion within the complex microchannel network meets the demands for the sandwich immunoassay and other complex microchip operations.

After demonstrating the precise microengine movement within the microchannel network, the microtransporters were functionalized with the corresponding antibody receptors to carry out the microchip immunoassays. In the first bioassay format, recognition of the target proteins was performed by functionalizing the microtransporters with the antibody via EDC/NHS chemistry (Fig. 1, IA), followed by the detection of the target protein, which is conjugated to a secondary antibody functionalized with streptavidin-coated polystyrene particles (SPP) (Fig. 1, IB). Therefore, the target binding event can be easily visualized by optical microscopy, as the antibody-modified microtransporter is efficiently picking-up the protein targetconjugated secondary antibody-coated S-PP.

The microtransporter functionalization protocol was optimized for efficient protein-antibody interaction and locomotion. A highly reproducible loading protocol was accomplished by the systematic optimization of the experimental conditions involved in the recognition and labeling steps. Several variables, such as the amount of microtransporters, vortex speed (during the antibody functionalization), concentration and incubation time of the antibodies, were thus examined and optimized, as summarized in Table S2 $\dagger$. The remaining amino moieties (after the modification) were blocked to minimize unspecific adsorption of the target proteins. ${ }^{21}$

The specific capture of the target protein by the antibodymodified microtransporters was first examined by using the LOC device. Fig. S2 and Video S1 $†$ illustrates an anti-IgGmodified microtransporter leaving the microengines reservoir of a linear-shaped chip (a), passing through the interconnection channel (b) and arriving to the second reservoir (c), containing the IgG/anti-IgG-modified biotinylated S-PP. The modified microtransporter thus navigates into this second reservoir, capturing the S-PP-tagged-IgG and leaving the reservoir (d). Upon returning to the channel, the microengine-loaded with
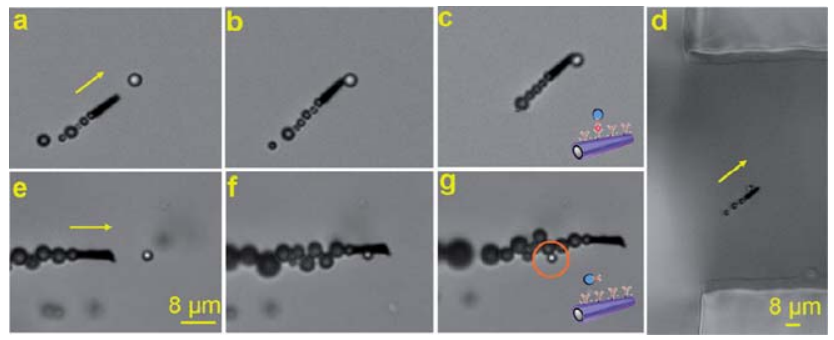

Fig. 3 (a-d) Anti-IgG-modified microengines approaching, contacting and capturing the IgG-antibody-modified particle complex. (e-g) Anti-lgG-modified microengines approaching, contacting but not capturing the antibody-modified particle (no IgG present; S-PP highlighted by an orange circle). It must be pointed out that the anti-IgG-modified S-PP is not pre-incubated with the IgG. All negative "controls" were performed on the surface of a glass slide. 
the tagged analyte-captures and transport three additional S-PPtagged-proteins (e). This fact demonstrates the strong affinity of the antigen-antibody interaction and the high towing force of the modified microtransporter. Fig. 3a-d and Video S $3 \dagger$ shows the pick-up and transport of a single antigen-conjugated microsphere by the anti-IgG-modified microtransporter. This figure illustrates the functionalized motor approaching (a), contacting (b) and capturing and transporting (c) the antigencoated S-PP within the microchip reservoir, followed by its entrance into a microchannel (d). The video shows the continuous transport of the protein-particle complex while crossing the entire reservoir ( $5 \mathrm{~mm}$ external diameter) at a speed of $44 \mu \mathrm{m} \mathrm{s}^{-1}$, which is $50 \%$ slower compared to that observed without the complex. In contrast, no pickup and transport are observed in the control experiment of Fig. 3e-g using the antibody-modified microtransporter and antibody-modified particle (in absence of the IgG antigen), despite the multiple direct motor-particle contacts.

Other control experiments are illustrated in ESI, Fig. S2 and Video S4 $\dagger$ (negative control 2). The modified microengine is shown first contacting but not capturing the unmodified PP (negative control 2; smaller size), while it is picking and transporting the IgG-B-anti-IgG-modified S-PP complex (acting as "positive control", bigger size). These results demonstrate that the antibody-modified microtransporters are able to specifically capture the target protein, indicating the great potential of these immuno-microengines for transporting and isolating proteins in a LOC device. The different size of these tagged and untagged particles $(\sim 2.0 \mu \mathrm{m} v s .0 .85 \mu \mathrm{m}$, respectively), allows a clear optical real-time visualization and discrimination between the "positive" and "negative controls" during the motor navigation in one single experiment. The similar size scale of the microengines and tags facilitates the clear visualization of the binding events in real time (see Fig. 3 and S2 and Videos S2$\mathrm{S} 4 \dagger$ ). This selective and rapid capture mechanism is attributed to the nearly instantaneous recognition of the proteins by the antibody-modified microengine (Fig. 3a-c). The localized fluid convection associated with the microengine vortex effect leads to a fast protein binding event, as was demonstrated for other bioisolation strategies based on such machines. ${ }^{23}$ Thus, unlike common immunoassays that require long incubation times $(15-30 \mathrm{~min})^{2,8}$ the microengine-induced localized convection dramatically accelerates the binding process.

Additional control experiments were performed by using different assay formats to demonstrate that the surfaceconfined antibody is solely responsible for the selective protein isolation (see the ESI methods section for details). For example, Fig. S3(A,a) and Video S4 $\dagger$ (negative control 3) demonstrate that an unmodified "control" microengine (without the immobilized antibody) do not capture the IgG-B-anti-IgG-modified S-PP complex, even after multiple direct contacts. Furthermore, no capture of the particle-protein complex was observed for microtransporters without $\mathrm{COOH}$ groups (on the outermost polymeric surface) that were incubated with the antibody (Fig. S3(A,b)) and Video S4 $\dagger$ (negative control 4).

An 'on-the-fly' DASA assay of the protein mixture was also carried out to confirm the high specificity of the
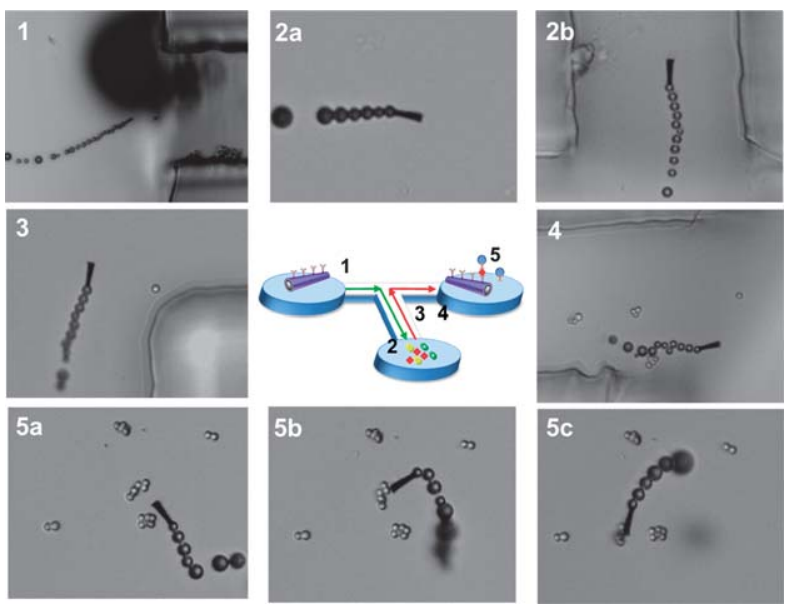

Fig. 4 'On-the-fly' microchip sandwich immunoassay: capture and transport proteins by using a DASA format. Microtransporters are modified with anti-lgG antibody and placed in a reservoir; S-PP are modified with the anti-IgG antibody and placed in another reservoir of the T-shaped LOC device; the free target IgG protein along with a 10 -fold excess $\left(\mu \mathrm{g} \mathrm{ml}^{-1}\right.$ ) of BSA and lysozyme are added into the third reservoir. A modified microtransporter moves from the modifiedmicroengine reservoir ( 1 ) to the one containing the protein mixture (2a) and after 10 min navigation is removed from the sample reservoir $3(2 b)$ to the anti-lgG SPP reservoir (4). In this last compartment the protein target, confined on the modified microtransporter, captures the modified particles $(5 a-c)$

surface-confined antibody towards the target protein. In such microchip sandwich immunoassay the target protein is first captured from a mixture of proteins by the anti-IgG modified microtransporters and later tagged with the S-PP tracer conjugated to the anti-IgG secondary antibody. The antibody-functionalized motors and spheres were placed in two different reservoirs of the T-shaped LOC device, with the third reservoir containing the $750 \mu \mathrm{g} \mathrm{ml} \mathrm{m}^{-1}$ of free IgG protein target along with a 10-fold excess of two other proteins, i.e., BSA and lysozyme (Fig. 4). A modified microtransporter thus travels from the modified-microengine reservoir (Fig. 4, 1) to the sample reservoir containing the protein mixture (Fig. 4, 2a); following a 10 min incubation while the microengine is continuously swimming, it departs from the sample reservoir (Fig. 4, 2b and 3) towards to the anti-IgG antibody reservoir (Fig. 4, 4). In this last compartment, the captured target protein (on the microtransporter) binds to the secondary antibody-modified particle (Fig. 4 and Video S5†), hence completing the microchip sandwich assay. These data clearly indicate the specific capture and transport of the target protein, reflecting the high affinity of the microtransporter surface antibody by the target protein and the effective minimization of non-specific binding due to the blocking of the remaining surface amino moieties after the antibody functionalization. In addition, the peroxide fuel and $\mathrm{NaCh}$ surfactant, essential for the microengine movement, do not compromise the specific protein-antibody interaction or the integrity of the bioreceptor coating.

The experimental reproducibility was investigated by using 8 different batches of the modified microtransporters following the identical processing steps. All the modified microtransporters were able to rapidly capture the S-PP-taggedtargets, most often immediately, upon few contacts. This 
indicates that different batches of modified transporters display a high protein capture efficiency and demonstrates the reliability of the fabrication, modification and movement steps involved in the entire process. The fast recognition and binding of the protein target indicates considerable promise for the rapid, direct and real time isolation of proteins.

Direct protein detection was tested by examining the ability of the antibody-modified microengines to recognize different quantities of the IgG target in the presence of a 10-fold excess of BSA and lysozyme. Capture and transport of the IgG was possible over the entire IgG concentration examined, i.e. from 20 to $750 \mu \mathrm{g} \mathrm{ml}^{-1}$. Fig. S4 and the corresponding Video S6 clearly illustrate that the functionalized microengines display an immediate 'on the fly' protein capture upon contacting the tagged-antigen present at the $20 \mu \mathrm{g} \mathrm{ml}^{-1}$ level, in the presence of a 10-fold excess of BSA and lysozyme proteins.

The practical application of the new immunomicrotransporter microchip approach was demonstrated also by the label-free detection of Staphylococcus aureus target bacteria (containing proteinA in its cell wall). ${ }^{29}$ As illustrated in Fig. 5 and Video $\mathrm{S} 7, \dagger$ an anti-proteinA antibody-modified microengine is able to recognize proteinA from the cell wall of Staphylococcus aureus ( $S$. aureus) while moving within the microchip. Fig. $5 \mathrm{a}-\mathrm{c}$ shows the modified microengine picking-up and loading this small, round, Gram-positive UTI-related bacteria and further leaving the reservoir containing the bacteria (Fig. 5d). The bacteria dimension (around $2 \mu \mathrm{m}$ ) offers convenient direct optical visualization of the binding event. The specific binding of the anti-proteinA-modified microengines to $S$. aureus was also examined in PBS containing $S$. aureus target bacteria along with a 5 -fold excess Saccharomyces cerevisiae (S. cerevisiae), a yeast specie frequently responsible for yeast infections and UTIs. ESI, Fig. S5a-c and Video S7† demonstrate the selective binding and transport of the small rod-shaped ( $\sim 2 \mu \mathrm{m}$ length) $S$. aureus bacteria (delineated by green dotted circles). In contrast, the modified microengine does not capture the larger round-shaped $S$. cerevisiae cells $(\sim 5 \mu \mathrm{m}$ in diameter) even when multiple contacts occur (delineated by red dotted circles in the video and ESI, $\uparrow$ Fig. S5d-g). The distinctly different size of the target $S$. aureus and the $S$. cerevisiae control $(\sim 2 \mu \mathrm{m}$ vs. $5 \mu \mathrm{m}$, respectively), allows a clear optical visualization of the selective capture of the target cell and discrimination against non-target

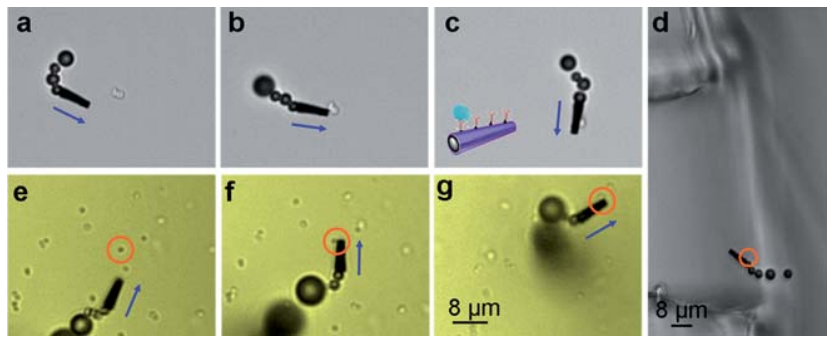

Fig. 5 Anti-ProtA-modified microtransporter approaching, contacting and loading $S$. aureus cells within a LOC device containing PBS (a-d), and in a human urine media containing bacteria $(\mathrm{e}-\mathrm{g}$ ), (experiments, on the surface of a glass slide). The target bacteria is highlighted by orange circles. cells during the microengine movement (see Video S8†). Note again that the similar size scale of the microengine and bacteria facilitates such real-time visualization of the binding event (see ESI, Fig. S5a-c and Video S8†). Such selective and rapid capture event is attributed to the nearly instantaneous recognition of the ProteinA molecules on the bacterial cell wall by the antiproteinA-modified microengine (ESI, Fig. S4a-c $\dagger$ ). Finally, capture and transport of the bacteria in an inoculated human urine samples was also demonstrated, as shown in Fig. 5e-g and ESI, Video S9.†

\section{Conclusions}

We have demonstrated the first example of microchip immunoassay protocols based on the movement of antibody-functionalized synthetic microtransporters within microchannel networks. New PEDOT:PEDOT-COOH/Ni/Pt immuno-microengines have thus been used for an 'on-the-fly' microchip DASA involving antibody-functionalized microtransporters including their directed capture and transport of target proteins along predetermined paths in the LOC system. Compared to common ELISA bioassays (in multiple well plates) the new motor-based immunoassay allows replacement of the common washing steps. Movement of the micrometer-size captured bacteria or sphere tag thus offer convenient direct real-time optical visualization of the protein binding event. Using different sizes of the microsphere tracers opens up the possibility of developing powerful chip-based multiplexed immunoassays involving different antibody-functionalized microtransporters. Such autonomous transport of antibody-functionalized nanomotors to 'on-the-fly' capture and isolate target proteins obviates the need for time-consuming and laborious multiple wash steps, hence greatly simplifying and accelerating the whole immunoassay protocol. Whenever needed, common regeneration procedures involving selected elution solutions, ${ }^{30,31}$ can be used for releasing the captured antigen and re-using the microengine. These results demonstrate the great potential of the antibody-modified microengines for the selective recognition, loading and isolation of target proteins and cells in a microchip device. Self-propelled immuno-microengines are expected to lead to new LOC bioanalytical microsystems with attractive protein isolation and detection capabilities, thus offering numerous opportunities in diverse areas, ranging from medical diagnostics to food safety.

\section{Experimental section}

\section{Synthesis of multilayer microengines}

The multilayer microtubes were prepared using a common template directed electrodeposition protocol. ${ }^{28}$ A cyclopore polycarbonate membrane, containing $2 \mu \mathrm{m}$ maximum diameter conical-shaped micropores (Catalog no. 7060-2511; Whatman, Maidstone, UK), was employed as a template. A $75 \mathrm{~nm}$ gold film was first sputtered on one side of the porous membrane to serve as a working electrode using a Denton Discovery 18. Sputtering was performed at room temperature under vacuum of $5 \times 10^{-6}$ Torr, DC power $200 \mathrm{~W}$ and Ar was flowed at $3.1 \mathrm{mT}$. Rotation 
speed was $65 \mathrm{rpm}$ along with a sputtering time of $90 \mathrm{~s}$. A Pt wire and an $\mathrm{Ag} / \mathrm{AgCl}$ with $3 \mathrm{M} \mathrm{KCl}$ were used as counter and reference electrodes, respectively. The membrane was then assembled in a plating cell with an aluminum foil serving as contact for the working electrode. PEDOT/PEDOT-COOH microtubes were electropolymerized up to $0.5 \mathrm{C}$ at $+0.85 \mathrm{~V}$ from a plating solution containing $7.5 \mathrm{mM}$ of each monomer (EDOT and EDOT-COOH), $100 \mathrm{mM}$ SDS and $7.5 \mathrm{mM} \mathrm{KNO}_{3}$, all of them were prepared from Sigma-Aldrich reagents. Then, the metallic layers were deposited from a Pt-Ni mixture solution. The first Pt layer was deposited galvanostatically at $-2 \mathrm{~mA}$ for $500 \mathrm{~s}$ to provide a smooth and high conductive surface after the polymer deposition and also improve the deposition of the next metallic layers. Afterwards the intermediate Ni layer was deposited amperometrically at $-1.3 \mathrm{~V}$ for $4.0 \mathrm{C}$ to achieve the magnetic properties that allow the micromotor guidance by properly orienting the magnetic field created by a simple neodymium magnet. Finally the catalytic inner Pt layer was deposited galvanostatically at $-2 \mathrm{~mA}$ for $450 \mathrm{~s}$. The Pt-Ni mixture solution was prepared by mixing the same volume of a commercial platinum solution (Platinum RTP; Technic Inc, Anaheim, CA) and a Nickel solution containing a mixture of $20 \mathrm{~g} \mathrm{l}^{-1} \mathrm{NiCl}_{2} \cdot 6 \mathrm{H}_{2} \mathrm{O}, 515 \mathrm{~g} \mathrm{l}^{-1}$ $\mathrm{Ni}\left(\mathrm{H}_{2} \mathrm{NSO}_{3}\right)_{2} \cdot 4 \mathrm{H}_{2} \mathrm{O}$, and $20 \mathrm{~g} \mathrm{l}^{-1} \mathrm{H}_{3} \mathrm{BO}_{3}$.

To release the microengines from the template, the sputtered gold layer was completely removed by mechanical hand polishing with 3-4 $\mu \mathrm{m}$ alumina slurry. The membrane was then dissolved in methylene chloride for $10 \mathrm{~min}$ to completely release the microtubes. Finally, microengines were washed repeatedly with methylene chloride, followed by ethanol and ultrapure water $(18.2 \mathrm{M} \Omega \mathrm{cm})$, three times of each, and collected by centrifugation at $6000 \mathrm{rpm}$ for $3 \mathrm{~min}$ after each wash.

\section{Microengines functionalization}

1-Ethyl-3-(3-dimethylaminopropyl) carbodiimide (EDC) $/ N$ hydroxysuccinimide (NHS) chemistry was used to activate the carboxyl-terminated groups from the polymer for conjugation with anti-Human IgG protein. For this purpose microengines were treated with $200 \mu \mathrm{l}$ of a 0.1 M MES buffer solution pH 5.0 containing $20 \mathrm{mg}$ EDC and $20 \mathrm{mg}$ NHS for $15 \mathrm{~min}$, washed with MES buffer 1 min and incubated with anti-Human IgG in PBS $1 \times \mathrm{pH} 7.2$ for $2 \mathrm{~h}$. The excess of antibody were washed in PBS buffer $(1 \times)$ pH 7.2 containing $0.05 \%$ of Tween 20 . The remaining amine reactive-esters from the activated carboxylic groups were blocked with $1 \mathrm{M}$ ethanolamine solution, $\mathrm{pH} 8.5$, for $30 \mathrm{~min}$ and BSA $1 \%$ for 1 hour with a washing step in between in PBS $1 \times \mathrm{pH}$ 7.2. In all the washing steps the microengines were centrifuged at $6000 \mathrm{rpm}$ for $4 \mathrm{~min}$. All the experiments were carried out under shaking at room temperature.

\section{Streptavidin polystyrene particles}

$20 \mu \mathrm{l}$ of streptavidin polystyrene particles were washed twice with B\&W buffer and then incubated in $50 \mu \mathrm{l}$ of $400 \mu \mathrm{g} \mathrm{ml} \mathrm{m}^{-1}$ biotin-anti-Human IgG antibody solution, for $15 \mathrm{~min}$. The excess of reagents was washed with Milli-Q water and particles incubated with $50 \mu \mathrm{l} \mathrm{IgG} \mathrm{of} 750 \mu \mathrm{g} \mathrm{ml}^{-1} \mathrm{IgG}$ antigen solution, for $15 \mathrm{~min}$. Finally the particles were washed with PBS buffer
$(1 \times) \mathrm{pH} 7.2$ containing $0.05 \%$ of Tween 20 and then resuspended in $20 \mu \mathrm{l}$ PBS buffer $(1 \times)$ for analysis.

\section{Equipment}

Template electrochemical deposition of microtube was carried out with a CHI 661D potentiostat (CH Instruments, Austin, TX). An inverted optical microscope (Nikon Eclipse Instrument Inc. Ti-S/L100), coupled with a $20 \times$ objective, along with a Hamamatsu digital camera C11440 and a NIS-Elements AR 3.2 software, were used for capturing movies at a frame rate of 20 frames per second. The speed of the microengines was tracked using a NIS-Elements tracking module and the results were statistically analyzed by using Origin software.

\section{Experimental procedure}

Self-propelled catalytic microengines in the microchannel were operated by filling the reservoirs and microchannel with a navigating solution consisting of a $2 \% \mathrm{NaCh} / 1 \% \mathrm{H}_{2} \mathrm{O}_{2}$ mixture in PBS buffer solution ( $\mathrm{pH}$ 7.2). Subsequently, microengines and particles were added into the reservoirs and were equilibrated until quiescent conditions were obtained. The movement of the microengines and all tasks were captured using the inverted optical microscope. Small quantities of either surfactant or fuel were further added when necessary. Such addition ensures not only the autonomous navigation of the microengines over prolonged time periods but also the absence of solution flow during the experiments (by maintaining the volume of mixture contained in the reservoirs).

\section{Acknowledgements}

This work was supported by the U.S. DTRA Grant HDTRA1-13-10002 and NATO Science for Peace and Security Program (SfP 983807). M. G. G., J.O. and M. G. acknowledge financial support from the University of Alcalá (Madrid), the Government of Catalonia and the Spanish MICINN, respectively. W.G. is a Howard Hughes Medical Institute International Student Research fellow. We also thank Allan Cortés and Ashley Pourazary for their support in the experiments.

\section{Notes and references}

1 S. R. Weinberger, T. S. Morris and M. Pawlak, Pharmacogenomics, 2000, 1, 395.

2 M. Vandermeeren, M. Mercken, E. Vanmechelen, J. Six, A. Van de Voorde, J. Martin and P. Cras, J. Neurochem., 1993, 61, 1828.

3 R. M. Lequin, Clin. Chem., 2005, 51, 2415.

4 N. H. Chiem and D. J. Harrison, Clin. Chem., 1998, 44, 591.

5 J. Wang, A. Ibáñez, M. P. Chatrathi and A. Escarpa, Anal. Chem., 2001, 73, 5323.

6 A. G. Crevillen, M. Hervás, M. A. López, M. C. González and A. Escarpa, Talanta, 2007, 74, 342.

7 A. E. Herr, A. V. Hatch, D. J. Throckmorton, H. M. Tran, J. S. Brennan, W. V. Giannobile and A. K. Singh, Proc. Natl. Acad. Sci. U. S. A., 2007, 104, 5268. 
8 J. M. Nam, C. S. Thaxton and C. A. Mirkin, Science, 2003, 301, 1884.

9 G. D. Bachand, H. Hess, B. Ratna, P. Satird and V. Vogel, Lab Chip, 2009, 9, 1661.

10 C. T. Lin, M. T. Kao, K. Kurabayashi and E. Meyhofer, Nano Lett., 2008, 8, 1041.

11 T. Fischer, A. Agarwal and H. Hess, Nat. Nanotechnol., 2009, 4, 162.

12 T. E. Mallouk and A. Sen, Sci. Am., 2009, 5, 72.

13 T. Mirkovic, N. S. Zacharia, G. D. Scholes and G. A. Ozin, ACS Nano, 2010, 4, 1782.

14 J. Wang, ACS Nano, 2009, 3, 4.

15 T. R. Kline, W. F. Paxton, T. E. Mallouk and A. Sen, Angew. Chem., Int. Ed., 2005, 117, 754.

16 Y. F. Mei, A. A. Solovev, S. Sanchez and O. G. Schmidt, Chem. Soc. Rev., 2011, 40, 2109.

17 S. Sanchez and M. Pumera, Chem.-Asian J., 2009, 4, 1402.

18 J. Wang, Lab Chip, 2012, 12, 1944.

19 J. Burdick, R. Laocharoensuk, P. M. Wheat, J. D. Posner and J. Wang, J. Am. Chem. Soc., 2008, 130, 8164.

20 S. Sanchez, A. A. Solovev, S. M. Harazim and O. G. Schmidt, J. Am. Chem. Soc., 2011, 133, 701.

21 S. Campuzano, D. Kagan, J. Orozco and J. Wang, Analyst, 2011, 136, 4621.
22 S. Balasubramanian, D. Kagan, C. J. Hu, S. Campuzano, M. J. Lobo-Castaño, N. Lim, D. Y. Kang, M. Zimmerman, L. Zhang and J. Wang, Angew. Chem., Int. Ed., 2011, 50, 4161.

23 D. Kagan, S. Campuzano, S. Balasubramanian, F. Kuralay, G. Flechsig and J. Wang, Nano Lett., 2011, 11, 2083.

24 J. Orozco, S. Campuzano, D. Kagan, M. Zhou, W. Gao and J. Wang, Anal. Chem., 2011, 83, 7962.

25 S. Campuzano, J. Orozco, D. Kagan, M. Guix, W. Gao, S. Sattayasamitsathit, J. C. Claussen, A. Merkoçi and J. Wang, Nano Lett., 2012, 12, 396.

26 M. Guix, J. Orozco, M. García, W. Gao, S. Sattayasamitsathit, A. A. Merkoçi, A. Escarpa and J. Wang, ACS Nano, 2012, 6, 4445.

27 W. Gao, S. Sattayasamitsathit, J. Orozco and J. Wang, J. Am. Chem. Soc., 2011, 133, 11862.

28 W. Gao, S. Sattayasamitsathit, A. Uygun, A. Pei, A. Ponedal and J. Wang, Nanoscale, 2012, 4, 2447.

29 B. Esteban-Fernández de Ávila, M. Pedrero, S. Campuzano, V. Escamilla-Gómez and J. M. Pingarrón, Anal. Bioanal. Chem., 2012, 403, 917.

30 R. Pei, X. Cui, X. Yang and E. Wang, Talanta, 2000, 53, 481.

31 J. J. Gilligen, P. Schuck and A. L. Yergey, Anal. Chem., 2002, 74, 2041. 